OLIVEIRA, L. C.; COSTA, E.; OLIVEIRA, A. D.; JORGE, M. H. A. Emergência do baruzeiro sob ambientes protegidos e substratos. Revista de Agricultura Neotropical, Cassilândia-MS, v. 1, n. 1, p. 10-16, jul./set. 2014.

\title{
EMERGÊNCIA DO BARUZEIRO SOB AMBIENTES PROTEGIDOS E SUBSTRATOS
}

\section{LETÍCIA CAROLINA DE OLIVEIRA ${ }^{1}$, EDILSON COSTA ${ }^{2}$, ANAIR DINIZ DE OLIVEIRA $^{1}$, MARÇAL HENRIQUE AMICI JORGE ${ }^{3}$}

\footnotetext{
1 UEMS, Aquidauana, MS, leticia_agroaqui@hotmail.com, anairdinizoliveira@gmail.com. ${ }^{2}$ UEMS/Cassilândia, MS, mestrine@uems.br; ${ }^{3}$ Embrapa Hortaliças, Brasília, DF, marcal.jorge@embrapa.br
}

RESUMO: O baruzeiro (Dipteryx alata Vog.), espécie arbórea de distribuição irregular na paisagem do Cerrado, apresenta diversos usos, tanto a planta como os frutos. O trabalho teve como objetivo a avaliação de ambientes protegidos e substratos na emergência de plântulas de Baru, na Universidade Estadual de Mato Grosso do Sul, Aquidauana-MS. Foram utilizados dois ambientes protegidos: estufa agrícola com tela termo-refletora sob o filme de polietileno e telado agrícola de tela preta (Sombrite ${ }^{\circledR}$ ). Foram utilizados 15 diferentes substratos das misturas de esterco bovino, húmus, ramas de mandioca e vermiculita. Cada ambiente foi considerado um experimento onde foi adotado o delineamento inteiramente casualizado e posteriormente foi realizada a análise conjunta dos mesmos. Substratos com menor porcentagem de matéria orgânica são mais favoráveis à emergência do baruzeiro. Maiores quantidades de vermiculita e ramas de mandioca, componentes físicos e materiais inertes, são mais importantes nos processos de emergência do baruzeiro. Os ambientes de cultivo não influenciaram a emergência do baruzeiro.

PALAVRAS-CHAVE: Dipteryx alata, húmus de minhoca, vermiculita, esterco bovino.

\section{BARUZEIRO EMERGENCE UNDER PROTECTED ENVIROMENTS AND SUBSTRATES}

\begin{abstract}
The baruzeiro plant and fruit (Dipteryx alata Vog.), tree species with irregular distribution in the Cerrado landscape, have many uses. This study aimed to evaluate protected environments and substrates on the Baruzeiro seedling emergence in the State University of Mato Grosso do Sul, in Aquidauana, Mato Grosso do Sul state. Two greenhouses were used: one with thermal-reflective screen under the polyethylene film and another only with black shading screen $\left(\right.$ Sombrite $\left.^{\circledR}\right)$. Fifteen substrates mixtures with cattle manure, earthworm compost, cassava stems and vermiculite were used. Initially, each protected environment was considered an experiment with a randomized design. Next, the environments were analyzed together. Substrates with lower percentage of organic matter are more favorable to baruzeiro seeding emergence. Greater amounts of vermiculite and cassava stems, physical components and inert materials, are more important in the baruzeiro seeding emergence processes. The cultivation environments did not influence the seeding emergence.
\end{abstract}

KEYWORDS: Dipteryx alata, earthworm compost, vermiculite, cattle manure.

\section{INTRODUÇÃO}

O Estado de Mato Grosso do Sul possui grande potencial para o desenvolvimento comercial de pomares de baruzeiro, pois além de apresentar matrizes nativas, possui grandes 
extensões de áreas disponíveis, sendo necessárias pesquisas em toda cadeia produtiva, iniciando-se pela formação de mudas de alta qualidade. A qualidade da muda é fundamental para implantação de pomares homogêneos.

O Baru apresenta grande quantidade de estoque de carbono, se mostrando ideal para os sistemas agroflorestais e para seqüestro de carbono (KURZATKOWSKI, 2007). Suas características rústicas, tanto do fruto, quanto da sua adaptação a diversos tipos de solos em especial com melhor drenagem, mostram o grande potencial de utilização em sistemas mais equilibrados ecologicamente (VERA; SOUZA, 2009).

Segundo Meletti (2000) e Pasqual et al. (2001) a exploração comercial de espécies geradoras de renda requerem mecanismos para a produção de mudas, buscando obter pomares uniformes. Dentre os fatores importantes para se obter mudas de qualidade, está o substrato, o qual, é um componente que possui elevada influência na produção de mudas, devendo-se dar especial atenção à escolha do mesmo.

Cada espécie de fruteira responde diferentemente ao tipo de substrato puro ou em misturas (FACHINELLO et al., 1995) e pesquisadores buscam identificar aqueles que promovem melhores condições e mudas de qualidade (MENEZES JÚNIOR; FERNANDES, 1999). Para o baruzeiro, Costa et al. (2012) verificaram que a vermiculita pura adubada, assim como substratos contendo $33,33 \%$ de esterco são indicados para formação de mudas de elevada qualidade.

A produção de mudas em ambientes protegidos promove melhor crescimento e desenvolvimento da planta e, consequentemente, melhores mudas para as produções a campo (CAVALCANTE et al., 2008). Costa et al. (2012) verificaram que os telados cobertos com tela termorrefletora e tela preta, ambos de 50\% de sombreamento, são mais indicados para a formação de mudas de baruzeiro que a estufa coberta com filme de polietileno de baixa densidade. Ajalla et al. (2012) observaram maiores biomassas radiculares em plantas de baru produzidas em telado de $50 \%$ comparados as produzidas em 0 e $30 \%$ de sombreamento, contudo essas porcentagens de sombreamento não diferiram para altura e diâmetro do colo. Mota et al. (2012) verificaram que níveis de sombreamento de 0, 50 e $70 \%$ não influenciaram na emergência das plântulas de baruzeiro.

Diante do exposto, o trabalho tem como objetivo a avaliação de ambientes protegidos e substratos na emergência de plântulas de Baru.

\section{MATERIAL E MÉTODOS}

Os experimentos, com a emergência de plântulas de Baru (Dipteryx alata Vogel), foram conduzidos na Unidade Universitária de Aquidauana, Universidade Estadual de Mato Grosso do Sul. A região possui uma altitude de $210 \mathrm{~m}$, longitude de $-55,67^{\circ}$ e latitude de 20,45 , e está situada na transição entre os biomas Cerrado e Pantanal. O experimento foi conduzido de 13 de abril a 06 de maio de 2012.

Foram utilizados dois ambientes protegidos: 1) estufa agrícola, modelo em arco, de estrutura em aço galvanizado, possuindo $8,00 \mathrm{~m}$ de largura por $18,00 \mathrm{~m}$ de comprimento, com altura sob a calha de $4,00 \mathrm{~m}$, coberta com filme polietileno de $150 \mu \mathrm{m}$ difusor de luz, com abertura zenital ao longo da cumeeira e fechamentos laterais e frontais com tela de monofilamento, malha para $50 \%$ de sombreamento (A1);2) telado agrícola, de estrutura em aço galvanizado, possuindo 8,00 $\mathrm{m}$ de largura por 18,00 $\mathrm{m}$ de comprimento e 3,50 $\mathrm{m}$ de altura, fechamento em $45^{\circ}$ de inclinação, com tela de monofilamento em toda sua extensão, malha com $50 \%$ de sombreamento $\left(\right.$ Sombrite $\left.^{\circledR}\right)$ (A2). 
No interior dos ambientes protegidos, para acondicionar as sementes, foi utilizado sacolas de polietileno $(15,0 \times 25,0 \mathrm{~cm})$, com capacidade de 1,6 litros, preenchidas com substratos oriundos das seguintes combinações: misturas de húmus de minhoca $(\mathrm{H})$, esterco bovino (E), vermiculita (V) e ramas de mandioca $(\mathrm{M})$, totalizando 15 substratos de diferentes proporções: $\mathrm{S} 1=25 \% \mathrm{H}+75 \% \mathrm{~V} ; \mathrm{S} 2=50 \% \mathrm{H}+50 \% \mathrm{~V} ; \mathrm{S} 3=75 \% \mathrm{H}+25 \% \mathrm{~V} ; \mathrm{S} 4=25 \% \mathrm{H}$ $+75 \% \mathrm{M} ; \mathrm{S} 5=50 \% \mathrm{H}+50 \% \mathrm{M} ; \mathrm{S} 6=75 \% \mathrm{H}+25 \% \mathrm{M} ; \mathrm{S} 7=25 \% \mathrm{E}+75 \% \mathrm{~V} ; \mathrm{S} 8=50 \% \mathrm{E}+$ $50 \% \mathrm{~V} ; \mathrm{S} 9=75 \% \mathrm{E}+25 \% \mathrm{~V} ; \mathrm{S} 10=25 \% \mathrm{E}+75 \% \mathrm{M} ; \mathrm{S} 11=50 \% \mathrm{E}+50 \% \mathrm{M} ; \mathrm{S} 12=75 \% \mathrm{E}$ $+25 \% \mathrm{M} ; \mathrm{S} 13=33,3 \% \mathrm{H}+33,3 \% \mathrm{E}+33,3 \% \mathrm{~V} ; \mathrm{S} 14=33,3 \% \mathrm{H}+33,3 \% \mathrm{E}+33,3 \% \mathrm{M} ; \mathrm{S} 15$ $=25 \% \mathrm{H}+25 \% \mathrm{E}+25 \% \mathrm{~V}+25 \% \mathrm{M}$. Foram realizadas análises física e química dos materiais orgânicos (Tabelas 1 e 2).

Tabela 1. Análise química dos materiais orgânicos dos substratos utilizados nos experimentos. Aquidauana - MS.

\begin{tabular}{|c|c|c|c|c|c|c|c|c|}
\hline & \multicolumn{8}{|c|}{ - } \\
\hline & $\mathrm{N}$ & $\mathrm{P}$ & $\mathrm{K}$ & $\mathrm{Ca}$ & $\mathrm{Mg}$ & $\mathrm{S}$ & $\mathrm{C}$ & $\mathrm{MO}$ \\
\hline $\mathrm{E}$ & 10,60 & 3,66 & 1,00 & 9,80 & 1,65 & 1,81 & 96,50 & 166,00 \\
\hline $\mathrm{H}$ & 14,80 & 4,46 & 1,00 & 26,70 & 12,50 & 3,53 & 163,00 & 281,00 \\
\hline \multirow[t]{3}{*}{ M } & 19,50 & 2,89 & 7,00 & 18,80 & 6,15 & 2,42 & 376,00 & 647,00 \\
\hline & - & - & - & $---\cdot-\cdot-$ & --------- & ----- $\mathrm{mg} \mathrm{k}$ & 1 & ------- \\
\hline & $\mathrm{pH}$ & $\mathrm{U}$ & $\mathrm{C} / \mathrm{N}$ & $\mathrm{Cu}$ & $\mathrm{Zn}$ & $\mathrm{Fe}$ & $\mathrm{Mn}$ & B \\
\hline $\mathrm{E}$ & 6,50 & 2,86 & 9,10 & 17,50 & 75,00 & 7800,00 & 310,00 & 11,47 \\
\hline $\mathrm{H}$ & 6,90 & 13,46 & 11,01 & 30,00 & 130,00 & 14800,00 & 370,00 & 14,40 \\
\hline $\mathrm{M}$ & 7,20 & 11,23 & 19,28 & 20,50 & 87,50 & 3440,00 & 520,00 & 20,70 \\
\hline
\end{tabular}

* Laboratório de análises do solo Solanalise, Cascavel-PR. $\mathrm{MO}=$ matéria orgânica; $\mathrm{U}=$ umidade em $\%$ a $65^{\circ} \mathrm{C} ; \mathrm{E}=\mathrm{esterco}$ bovino; $\mathrm{H}=$ húmus de minhoca; $\mathrm{M}$ = ramas de mandioca; $\mathrm{C} / \mathrm{N}=$ relação carbono e nitrogênio.

Tabela 2. Densidade Úmida (U) e Seca (S) dos substratos. Aquidauana - MS.

\begin{tabular}{|c|c|c|c|c|c|c|c|c|}
\hline \multicolumn{9}{|c|}{ Densidade $\left(\mathrm{kg} \cdot \mathrm{m}^{-3}\right)$} \\
\hline & $\mathrm{S} 1 * *$ & $\mathrm{~S} 2$ & S3 & S4 & S5 & S6 & S7 & S8 \\
\hline $\mathrm{U}^{*}$ & 791,11 & 958,74 & 1104,50 & 931,50 & 1146,17 & 1229,06 & 648,14 & 741,17 \\
\hline \multirow[t]{2}{*}{$\mathrm{S}$} & 279,83 & 479,71 & 500,62 & 273,65 & 502,11 & 648,20 & 209,47 & 270,36 \\
\hline & S9 & S10 & S11 & S12 & S13 & S14 & S15 & - \\
\hline $\mathrm{U}$ & 838,14 & 652,16 & 700,27 & 816,03 & 956,67 & 766,94 & 988,09 & - \\
\hline $\mathrm{S}$ & 399,47 & 216,58 & 244,08 & 376,33 & 442,97 & 289,88 & 457,02 & - \\
\hline $\begin{array}{l}* \mathrm{U}= \\
25 \% \\
+25\end{array}$ & 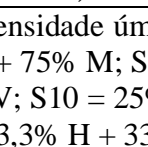 & & & & & & & \\
\hline
\end{tabular}

Foi utilizada uma semente por recipiente e a coleta dos dados de emergência foi realizada até a estabilização do tratamento mais veloz. Foi mensurado o índice de velocidade de emergência (IVE) proposto por Maguire (1962) e a porcentagem de emergência (PE) na estabilização, ou seja, quando as contagens do tratamento mais veloz se repetiram pela terceira vez.

Em cada ambiente protegido, os experimentos foram conduzidos em delineamento inteiramente casualizado, com cinco repetições de quatro plântulas por parcela. Foi utilizada a análise de grupos de experimentos. Para realizar esta análise, primeiramente, os quadrados médios dos resíduos das análises de variâncias individuais dos substratos dentro dos ambientes 
foram avaliados e quando estes não ultrapassaram a relação aproximada de 7:1 (BANZATTO; KRONKA, 2006), foi realizada a análise conjunta.

O esterco bovino foi compostado por 30 dias, homogeneizado e secado. O húmus de minhoca foi obtido de empresa comercial. As ramas de mandioca foram trituradas em moinho peneira número 08, secadas e, posteriormente, compostada por, aproximadamente, 60 dias. A vermiculita, obtida de empresa comercial, foi a de textura super fina. A irrigação foi realizada manualmente com o uso de regadores, duas vezes ao dia (pela manhã e à tarde), visando suprir as necessidades hídricas das mudas, procurando não encharcar os substratos e mantê-los em boas condições para o desenvolvimento radicular.

Os dados foram submetidos à análise de variância (teste F) e as médias comparadas pelo teste de Scott-Knott a 5\% de probabilidade para os substratos e pelo próprio teste F para os ambientes de cultivo, com o software Sisvar (FERREIRA, 2010).

\section{RESULTADOS E DISCUSSÃO}

A relação entre os quadrados médios do resíduo das análises de variâncias individuais não ultrapassou a relação de 7:1 (Tabela 3). Portanto, foi realizada a análise de grupos de experimentos para comparação dos ambientes de cultivo. Os substratos com $75 \%$ de húmus $(\mathrm{S} 3=75 \% \mathrm{H}+25 \% \mathrm{~V} ; \mathrm{S} 6=75 \% \mathrm{H}+25 \% \mathrm{M})$ e $75 \%$ de esterco $(\mathrm{S} 9=75 \% \mathrm{E}+25 \% \mathrm{~V})$ apresentaram os menores valores de índice de velocidade de emergência e menor porcentagem de emergência, diferindo significativamente dos demais tratamentos (Tabela 3). Provavelmente a elevada quantidade de matéria orgânica (75\%) tenha inibido a emergência do baruzeiro. Costa et al. (2012) também verificaram que substratos com elevada porcentagem de esterco bovino não foram favoráveis a emergência do baruzeiro. Santos et al. (2011) relatam que espécie nativa de cerrado, como por exemplo, o jatobazeiro-do-cerrado, é adaptada a solos mais ácidos, e emitem suas radículas em substrato com menor quantidade de matéria orgânica. $\mathrm{O}$ mesmo pode ter ocorrido com o baruzeiro no presente trabalho, assim, o húmus pode ter elevado o valor do $\mathrm{pH}$ e consequentemente agido negativamente na sua germinação.

Nos tratamentos que receberam maior quantidade de vermiculita tenderam a proporcionar maior índice de velocidade de emergência e maior porcentagem de emergência (Tabela 3). Tais observações coincidem com Costa et al. (2012) que verificaram melhor emergência de plântulas em vermiculita pura. Minami (1995) relata que o uso da vermiculita como componente do substrato se justifica por ser um mineral com alta capacidade de absorção de água, elevada porosidade, baixa densidade, elevada CTC, fatores desejáveis para garantir a qualidade do substrato. Cañizares et al. (2002) verificaram maior sobrevivência de mudas de pepineiro cultivados em vermiculita que em palha de arroz.

As maiores porcentagens de emergências (PE) foram verificadas nos substratos $\mathrm{S} 1=$ $25 \% \mathrm{H}+75 \% \mathrm{~V}, \mathrm{~S} 7=25 \% \mathrm{E}+75 \% \mathrm{~V}(82,50 \%)$ e $\mathrm{S} 10=25 \% \mathrm{E}+75 \% \mathrm{M}(85,00 \%), \mathrm{em}$ substratos com as menores quantidades de esterco e maiores quantidades de vermiculita e ramas de mandioca, revelando que os componentes físicos dos substratos (materiais inertes) são mais importantes nos processos de emergência do baruzeiro (Tabela 3). Estes valores de porcentagem de emergência foram superiores aos observados por Costa et al. (2012) que foi de $66,1 \%$ e Mota et al. (2012) de 75\%, ambos em telado de cor preta de $50 \%$ de sombreamento. 
Tabela 3. Índice de velocidade de emergência (IVE) e porcentagem de emergência (PE) do Baruzeiro.

\begin{tabular}{ccc}
\hline Estufa PEBD & $\begin{array}{c}\text { Índice de velocidade } \\
\text { de emergência (IVE) }\end{array}$ & $\begin{array}{c}\text { Porcentagem de } \\
\text { emergência (PE) }\end{array}$ \\
\hline Telado Preto & $1,38 \mathrm{a}$ & $65,16 \mathrm{a}$ \\
$\mathrm{S} 2=50 \% \mathrm{H}+50 \% \mathrm{~V}$ & $1,44 \mathrm{a}$ & $67,23 \mathrm{a}$ \\
$\mathrm{S} 3=75 \% \mathrm{H}+25 \% \mathrm{~V}$ & $1,76 \mathrm{~A}$ & $85,00 \mathrm{~A}$ \\
$\mathrm{~S} 4=25 \% \mathrm{H}+75 \% \mathrm{M}$ & $1,52 \mathrm{~A}$ & $69,30 \mathrm{~B}$ \\
$\mathrm{~S} 5=50 \% \mathrm{H}+50 \% \mathrm{M}$ & $0,91 \mathrm{C}$ & $37,50 \mathrm{C}$ \\
$\mathrm{S} 6=75 \% \mathrm{H}+25 \% \mathrm{M}$ & $1,52 \mathrm{~A}$ & $70,00 \mathrm{~B}$ \\
$\mathrm{~S} 7=25 \% \mathrm{E}+75 \% \mathrm{~V}$ & $1,42 \mathrm{~A}$ & $71,80 \mathrm{~B}$ \\
$\mathrm{~S} 8=50 \% \mathrm{E}+50 \% \mathrm{~V}$ & $0,72 \mathrm{C}$ & $47,50 \mathrm{C}$ \\
$\mathrm{S} 9=75 \% \mathrm{E}+25 \% \mathrm{~V}$ & $1,83 \mathrm{~A}$ & $82,50 \mathrm{~A}$ \\
$\mathrm{~S} 10=25 \% \mathrm{E}+75 \% \mathrm{M}$ & $1,26 \mathrm{~B}$ & $65,00 \mathrm{~B}$ \\
$\mathrm{~S} 11=50 \% \mathrm{E}+50 \% \mathrm{M}$ & $1,05 \mathrm{C}$ & $46,80 \mathrm{C}$ \\
$\mathrm{S} 12=75 \% \mathrm{E}+25 \% \mathrm{M}$ & $1,87 \mathrm{~A}$ & $85,00 \mathrm{~A}$ \\
$\mathrm{~S} 13=33,3 \% \mathrm{H}+33,3 \% \mathrm{E}+33,3 \% \mathrm{~V} ;$ & $1,31 \mathrm{~B}$ & $65,00 \mathrm{~B}$ \\
$\mathrm{~S} 14=33,3 \% \mathrm{H}+33,3 \% \mathrm{E}+33,3 \% \mathrm{M}$. & $1,26 \mathrm{~B}$ & $60,00 \mathrm{~B}$ \\
$\mathrm{~S} 15=25 \% \mathrm{H}+25 \% \mathrm{E}+25 \% \mathrm{~V}+25 \% \mathrm{M}$ & $1,56 \mathrm{~A}$ & $70,00 \mathrm{~B}$ \\
\hline $\mathrm{CV}$ & $1,77 \mathrm{~A}$ & $75,00 \mathrm{~B}$ \\
RQMR & $1,41 \mathrm{~A}$ & $62,50 \mathrm{~B}$ \\
\hline Fcal (ambientes) & 32,35 & 30,30 \\
Fcal (substratos) & 1,19 & 1,06 \\
Fcal (interações) & $0,471^{\mathrm{NS}}$ & $0,398^{\mathrm{NS}}$ \\
\hline
\end{tabular}

* Letras iguais minúsculas para os ambientes pelo teste $\mathrm{F}$ e letras iguais maiúsculas para os substratos pelo Teste de ScottKnott a 5\% de probabilidade não diferem entre si, para cada variável; $\mathrm{H}=$ húmus de minhoca; $\mathrm{V}=$ vermiculita; $\mathrm{E}=$ esterco bovino; $\mathrm{M}=$ ramas de mandioca triturada.; $\mathrm{CV}=$ coeficiente de variação; $\mathrm{RQMR}$ = relação entre o maior e o menor quadrado médio do resíduo das análises individuais dos substratos dentro dos ambientes de cultivo. Fcal = F calculado; * significativo a $5 \%$ de probabilidade; ** significativo a $1 \%$ de probabilidade; ${ }^{\text {NS }}$ não significativo.

Os substratos com menor quantidade de matéria orgânica, com $25 \%$ de húmus ou esterco, assim como os substratos com misturas de dois, três ou quatro materiais, foram mais favoráveis à emissão do hipocótilo e emissão da radícula do baruzeiro. Para estes substratos, com menor porcentagem de matéria orgânica, não foi verificada diferença significativa entre os ambientes de cultivo testados (Tabela 3). Ajalla et al. (2012) testando níveis de sombreamento de $0 \%, 30 \%$ e $50 \%$ na formação de mudas de baru também não observaram diferenças nas altura e diâmetros do colo nos ambientes testados, contudo maior massa seca das raízes foi encontrada no telado com 50\% de sombreamento. Mota et al. (2012) verificaram que os níveis de sombreamento 0,50 e $70 \%$ não influenciaram na emergência das plântulas de baruzeiro conforme observado no presente trabalho, entretanto, maiores plantas foram observadas em telado de $50 \%$ de sombreamento.

\section{CONCLUSÕES}

Substratos com menor porcentagem de matéria orgânica são mais favoráveis à emergência do baruzeiro.

Os ambientes de cultivo não influenciaram a emergência do baruzeiro para os substratos testados. 


\section{AGRADECIMENTOS}

À Fundação de Apoio ao Desenvolvimento do Ensino, Ciência e Tecnologia do Estado de Mato Grosso do Sul (FUNDECT), Proc. No 23/200.647/2012. À Universidade Estadual de Mato Grosso do Sul (UEMS), Unidade Universitária de Cassilândia (UUC). Ao Conselho Nacional de Desenvolvimento Científico e Tecnológico (CNPq), Proc. No 300829/2012-4.

\section{REFERÊNCIAS BIBLIOGRÁFICAS}

AJALLA, A. C. A.; VOLPE, E.; VIEIRA, M. C.; ZARATE, N. A. H. Produção de mudas de baru (Dipteryx alata Vog.) sob três níveis de sombreamento e quatro classes texturais de solo. Revista Brasileira de Fruticultura, Jaboticabal-SP, v. 34, n. 2, p. 888-896, 2012.

BANZATTO, D. A.; KRONKA, S. N. Experimentação agrícola. 4. ed. Jaboticabal-SP: FUNEP/UNESP, 2006. 237 p.

CAÑIZARES, K. A. L.; COSTA, P. C.; GOTO, R.; VIEIRA, A. R. M. Desenvolvimento de mudas de pepino em diferentes substratos com e sem uso de solução nutritiva. Horticultura Brasileira, Brasília, v. 20, n. 2, p. 227-229, 2002.

CAVAlCANTE, T. R. M.; NAVES, R. V.; SERAPHIN, J. C.; CARVALHO, G. D. Diferentes ambientes e substratos na formação de mudas de araticum. Revista Brasileira de fruticultura, Jaboticabal-SP, v. 30, n. 1, p.235-240, 2008.

COSTA, E.; OLIVEIRA, L. C.; SANTO, T. L. E.; LEAL, P. A. M. Production of baruzeiro seedling in different protected environments and substrates. Engenharia Agrícola, Jaboticabal-SP, v.32, n. 4, p. 633-641, 2012.

FACHINELlO, J. C.; HOFFMANN, A.; NACHTIGAL, J. C. Propagação de plantas frutíferas de clima temperado. 2. ed. Pelotas-RS: UFPel, 1995. 178 p.

FERREIRA, D. F. SISVAR - Sistema de análise de variância. Versão 5.3. Lavras-MG: UFLA, 2010.

KURZATKOWSKI, D. Potencial do seqüestro de carbono nos sistemas agroflorestais: analise dos quatro sistemas implantados no município de Pium-TO. Revista Carbono Social, Palmas-TO, v. 1, n. 4, p. 75-80, 2007.

MAGUIRE, J. D. Speed of germination aid in selection and evaluation of seedling emergence and vigor. Crop Science, Madison, v. 2, n. 2, p. 176-177, 1962.

MELETTI, L. M. M. Propagação de frutíferas tropicais. Guaíba-RS: Agropecuária, 2000. 239 p.

MENEZES JUNIOR, F. O. G.; FERNANDES, H. S. Efeitos de substratos formulados com esterco de curral e substratos comerciais na produção de mudas de alface. Revista Científica Rural, Bagé-RS, v. 4, n. 2, p. 15-23, 1999.

MINAMI, K. Produção de mudas em recipientes. In: MINAMI, K (Ed) Produção de mudas de alta qualidade em horticultura. São Paulo-SP: Fundação Salim Farah Maluf, 1995. cap. 3. p. 85-101.

MOTA L. H. S.; SCALON, S. P. Q.; HEINZ, R. Sombreamento na emergência de plântulas e no crescimento inicial de Dipteryx alata Vog. Ciência Florestal, Santa Maria-RS, v. 22, n. 3, p. 423-431, 2012. 
PASQUAL, M.; CHALFUn, N. N. J.; RAMOS, J. D.; VAlE, M. R.; SILVA, C. R. Fruticultura comercial: propagação de plantas frutíferas. Lavras-MG: UFLA/FAEPE, 2001. $137 \mathrm{p}$.

SANTOS, L. C. R.; COSTA, E.; LEAL, P. A. M.; NARDELlI, E. M. V.; SOUZA, G. S. A. Ambientes protegidos e substratos com doses de composto orgânico comercial e solo na formação de mudas de jatobazeiro em Aquidauana-MS. Engenharia Agrícola, JaboticabalSP, v. 31, n. 2, p. 249-259, 2011.

VERA, R.; SOUZA, E. R. B. Baru. Revista Brasileira de Fruticultura, Jaboticabal-SP, v.31, n. 1, p. 1, 2009. 